\title{
Rational-differential design of highly specific glycomimetic ligands: Targeting DC-SIGN and excluding Langerin recognition
}

Vanessa Porkolab ${ }^{\dagger}$, Eric Chabrol ${ }^{\dagger}$, Norbert Varga ${ }^{\S}$, Stefania Ordanini ${ }^{\S}$, Ieva Sutkevičiūté ${ }^{\dagger}$, Michel Thépaut $^{\dagger}$, M. José García-Jiménez ${ }^{\perp}$, Eric Girard ${ }^{\dagger}$, Pedro M. Nieto ${ }^{\perp}$, Anna Bernardi*\$,\#, Franck Fieschi* ${ }^{*}$.

$\dagger$ Univ. Grenoble Alpes, CNRS, CEA, Institut de Biologie Structurale, F-38044 Grenoble, France.

$\S$ Universita` degli Studi di Milano (UniMI), Dip. Chimica, via Golgi 19, 20133, Milano, Italy.

\# CNR-ISTM, Inst. of Molecular Science and Technologies, Milano, Italy.

${ }^{\perp}$ Glycosystems Laboratory, Instituto de Investigaciones Químicas (IIQ), Centro de Investigaciones Científicas Isla de La Cartuja, CSIC and Universidad de Sevilla, Américo Vespucio, 49, 41092 Sevilla, Spain.

\section{Graphical Table of Content}

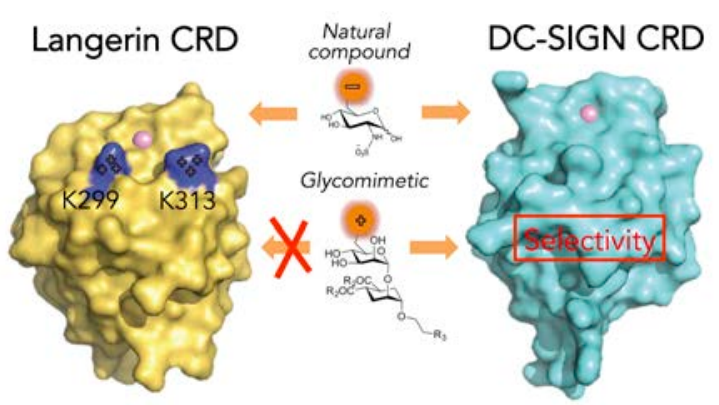


ABSTRACT: At the surface of dendritic cells, C-type lectin receptors (CLRs) allow the recognition of carbohydratebased PAMPS or DAMPS (pathogen- or danger-associated molecular patterns respectively) and promote immune response regulation. However, some CLRs are hijacked by viral and bacterial pathogens. Thus, the design of ligands able to target specifically one CLR, to either modulate a immune response or to inhibit a given infection mechanism, has a great potential value in therapeutic design. A case study is the selective blocking of DC-SIGN, involved notably in HIV trans-infection of $\mathrm{T}$ lymphocytes, without interfering with Langerin-mediated HIV clearance. This is a challenging task due to their overlapping carbohydrate specificity. Towards the rational design of DC-SIGN selective ligands, we performed a comparative affinity study between DC-SIGN and Langerin with natural ligands. We found that GlcNAc is recognized by both CLRs, however, selective sulfatations are shown to increase the selectivity in favour of Langerin. With the combination of site-directed mutagenesis and X-ray structural analysis of Langerin/GlcNS6S complex, we highlighted that 6-sulfatation of the carbohydrate ligand induced Langerin specificity. Additionally, the K313 residue from Langerin was identified as a critical feature of its binding site. Using a rational and a differential approach in the study of CLR binding sites, we designed, synthetized and characterized a new glycomimetic which is highly specific for DC-SIGN vs Langerin. STD NMR, SPR and ITC characterizations show that compound 7 conserved the overall binding mode of the natural disaccharide while possessing an improved affinity and a strict specificity for DC-SIGN

C-type lectin receptors (CLRs) are central sensing systems for the regulation of the initial immune response through dendritic cells. ${ }^{1,2}$ Together with the Toll-Like Receptor (TLR) family of pathogen recognition receptors (PRRs), they are key elements in the recognition of both pathogen-associated molecular patterns (PAMPs) and of danger-associated molecular patterns (DAMPs) displayed by altered cells. Dendritic cells use CLRs to recognize carbohydrate-based PAMPs or DAMPs in a $\mathrm{Ca}^{2+}$-dependent manner. Upon recognition, and depending on the crosstalk with TLRs, an activation or tolerance response will be promoted by dendritic cells towards specific T-cells. ${ }^{3,4}$ Apart from being essentials for immune regulation, some of these CLRs are also hijacked by pathogens during their infection process. For instance, LSECtin and DC-SIGN are used to this purpose respectively by Ebola virus and by a wide range of virus and bacterial pathogens (from HIV, M. tuberculosis to C. albicans for DC-SIGN). ${ }^{5-9}$ Thus, CLRs have become attractive targets for the design of new ligands able to modulate the immune response towards activation or inhibition as function of the CLRs addressed. Such ligands could become anti-infective agents in cases where CLRs are used by pathogens to infect the host.

The development of such ligands as molecular probes for the modulation of various CLRs is the subject of intense efforts. ${ }^{10-12}$ However, the development of highly specific and effective ligands towards CLRs faces several specific bottlenecks. The first problem is the nature of the natural ligands, oligosaccharides, that are easily metabolized and that may have a low level of bioavailability in therapeutic conditions. Second, natural carbohydrates frequently bind within CLRs' $\mathrm{Ca}^{2+}$ binding sites using multiple binding modes, thus making the specific benefit of any targeted modification almost unpredictable. Indeed, depending on the mode of binding, a given structural modification of a ligand may either improve or reduce and even eliminate the binding affinity. Third, the active sites of CLRs are shallow binding pockets and open to the solvent (Figure 1A), contributing to low affinity ligands, often in the $\mathrm{mM}$ range. And finally, designed ligands often generate off-target effects due to carbohydrate cross-recognition between various CLRs. Altogether, these barriers make the rational design of tightly bound and CLR-selective ligands difficult. Despite theses challenges, methods in carbohydrate chemistry and structure-based design have progressed, opening the door to successes in CLR ligand design. 

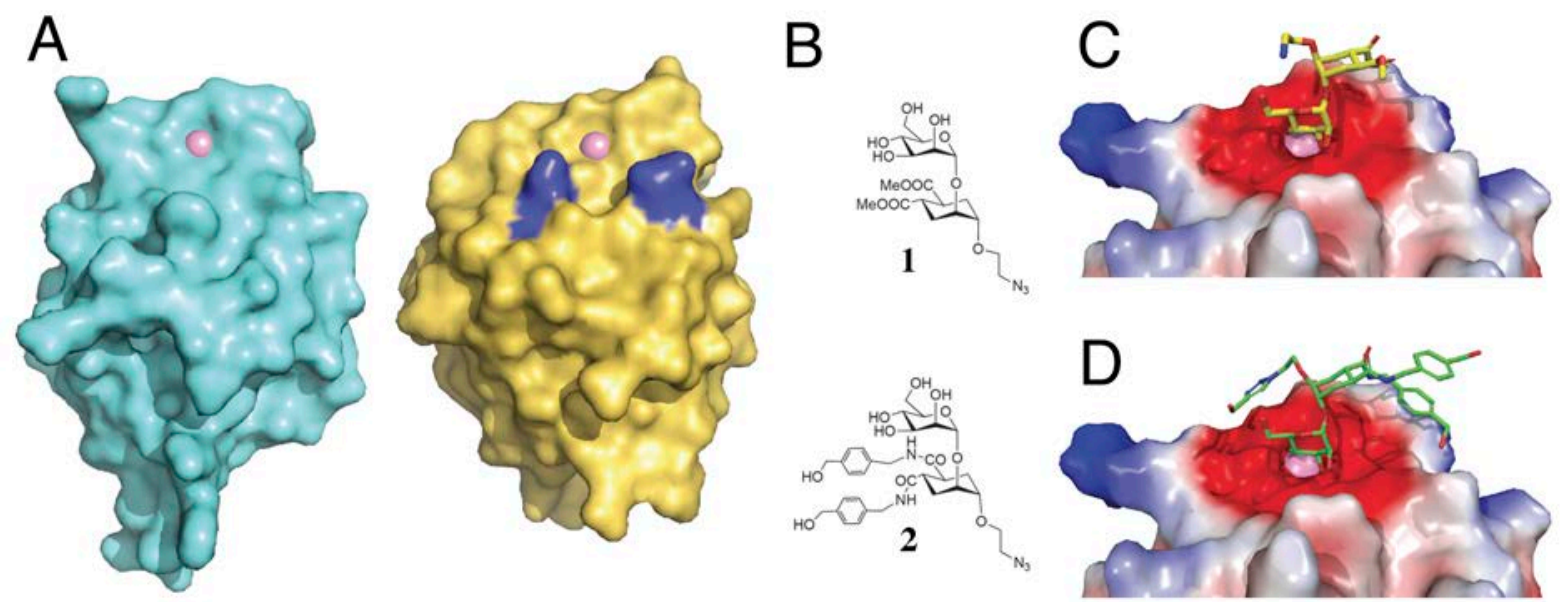

Figure 1. Structural comparison and glycomimetic design targeting the carbohydrate binding domain of DC-SIGN vs Langerin. (A) Surface representation of DC-SIGN CRD (cyan, pdb 2it6) and Langerin CRD (yellow, pdb 3c22). Ca ${ }^{2+}$ ions within the binding site are in pink. The two Langerin specific lysines, K299 and K313 are represented in dark blue. (B) Structure of two generations of glycomimetic directed against DC-SIGN. (C) and (D) Electrostatic surfaces of DC-SIGN CRD in complex with 1 (pdb 2xr5) and 2 (STD NMR and molecular docking), respectively.

DC-SIGN, one of the most investigated CLRs up to now, is a good example of the cumulative difficulties typically encountered with CLRs. To date, issues involving biostability and bioavailability, ${ }^{13-16}$ heterogeneous modes of binding with the $\mathrm{Ca}^{2+}$ binding site, and weak protein-carbohydrate affinity have been successfully addressed for DC-SIGN by several groups. ${ }^{13,17-19}$ Our previous work set out to rationally design glycosidase-resistant glycomimetic ligands that bound to DC-SIGN with binding modes similar to the natural ligands. From this approach, we identified two glycomimetics, a pseudo-di and a pseudo-trisaccharide, as lead compounds able to bind efficiently DC-SIGN and even to be effective in anti-HIV trans-infection test (Figure 1B). ${ }^{20-22}$ However, because of the unpredictable multiple binding mode of carbohydrates with CLRs, ${ }^{23-25}$ we have been able to establish that only the pseudo-disaccharide 1 meets the requirement of a unique binding mode within DC-SIGN (Figure 1C) and allows further rational molecule improvement.

Using structural information regarding the binding mode of this glycomimetic, ${ }^{23}$ we rationally improved the relative affinity of compound 1 for DC-SIGN by substituting the methyl ester groups on the cyclohexane moiety with the amides in compound $\mathbf{2}$. The extended ligand surface contact within the binding site leads to an improvement of its affinity. ${ }^{26}$ Other groups, also using structural information and molecular modeling, have been successful in affinity improvement of monovalent ligands. ${ }^{15,27,28}$ However, further affinity improvement in the low $\mu \mathrm{M}$ or even the $\mathrm{nM}$ ranges have been reached only using multivalent presentations of lead ligands on molecular scaffolds. ${ }^{18,29-31}$ On the other hand, the carbohydrate cross-recognition between different CLRs, has not been addressed specifically up to now. During the development of compound 2, we discovered a fortuitous selectivity increase in favor of DC-SIGN relative to Langerin, another CLRs also able to recognize HIV envelope glycoprotein, gp $120{ }^{23,26}$ However, even if some selectivity could be obtained, the remaining activity towards Langerin is not negligible particularly in multivalent presentation that will also improve this non-desired interaction. The case study of DC-SIGN and Langerin has a particular interest due to their opposite physiological consequences, respectively as promoting the T-lymphocytes infection or contributing to virus elimination. ${ }^{7,32-34}$ In addition, Langerin targets HIV to Birbeck granules, which are believed to be an atypical antigen processing pathways to Langerhans cells. ${ }^{32,35}$ For all these reasons, the design of specific and selective antagonists for DC-SIGN over Langerin is fully justified.

Here, in this work, we address specifically the unsolved problem of CLR cross-recognition. We pioneer a differential rational design approach by which we analyze the structural and functional variances between the two binding sites, 
developing ligands that favor DC-SIGN and disfavor recognition by Langerin. Differential rational design aims to reach a complete specificity for, DC-SIGN. Here, the DC-SIGN/Langerin pair is used as a case study for the potential applications in the design of HIV capture inhibitors and of immune regulation therapeutics.

\section{RESULTS AND DISCUSSION}

Sulfation-induced selectivity towards Langerin. The main difference in ligand recognition between DC-SIGN and Langerin lies in the ability of Langerin to specifically recognize sulfated glycans. In order to design ligand modifications to generate specific glycomimetic candidates, recognition of sulfated glycans by Langerin need to be understood. Langerin recognizes sulfated-glycans with terminal $6-\mathrm{SO}_{4}$-Gal residues ${ }^{36,37}$ or glycosaminoglycans containing sulfated derivatives of $N$-Acetyl-D-glucosamine (GlcNAc). ${ }^{38,39}$ Because GlcNAc and Man share the same 3,4-di-equatorial hydroxyl groups for binding to $\mathrm{Ca}^{2+}$, the binding mode of GlcNAc derivatives is expected to be similar to Man. Thus, we focused our selectivity characterization on GlcNAc-sulfated derivatives in order to compare and transfer newly identified features to the mannose-based glycomimetic lead compound $\mathbf{1}$.
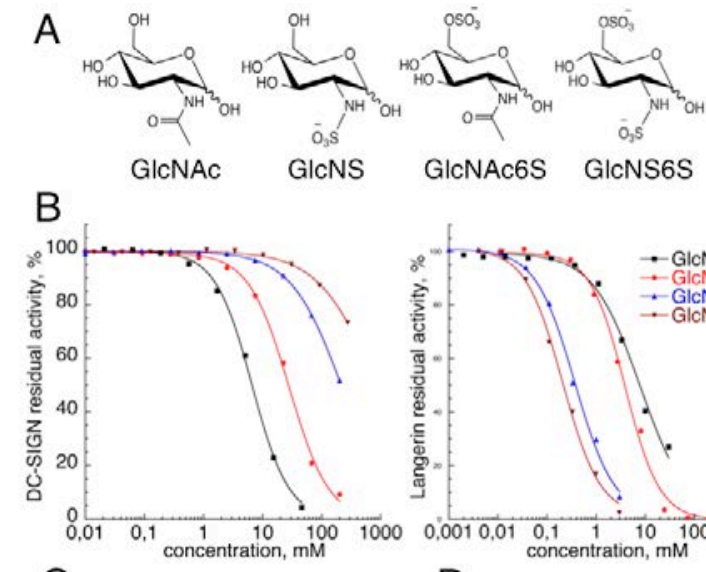

GlcNAc6S GlcNS6S
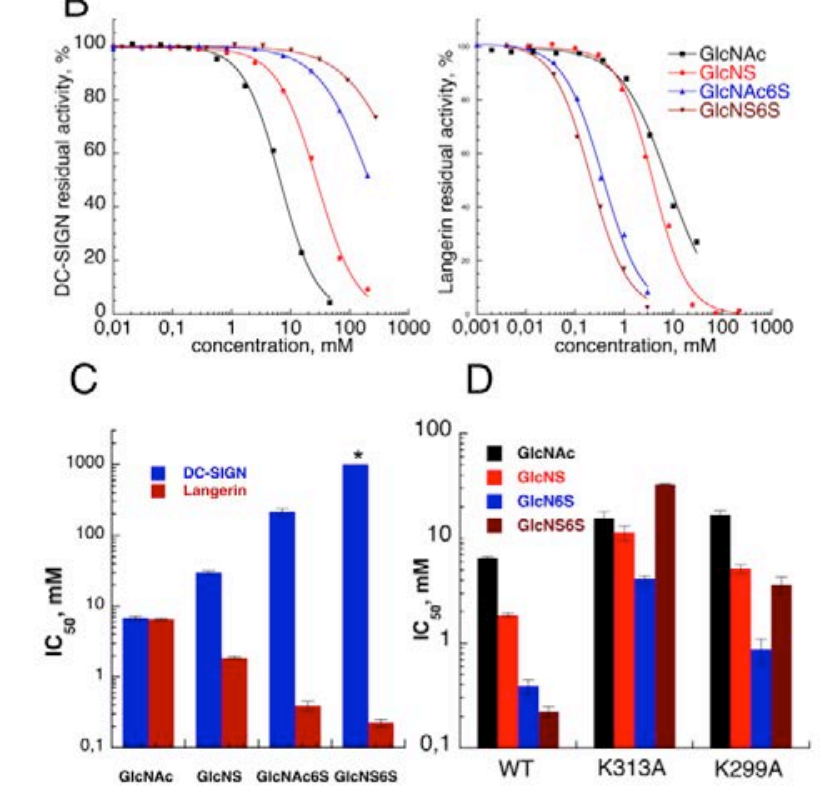

$\mathrm{D}$

Figure 2. Inhibition of DC-SIGN and Langerin binding to Man-BSA by sulfated derivatives of GlcNAc in a SPR competition assay. (A) Structure of GlcNAc-sulfated derivatives used in the study. (B) Inhibition curves for DC-SIGN (left) and Langerin (right). (C) Comparison of $\mathrm{IC}_{50}$, extracted from (B), for DC-SIGN (blue) or Langerin (red) as a function of the sulfatation degree and position. (D) Impact of lysine mutations in Langerin on the $\mathrm{IC}_{50}$ of $\mathrm{GlCNAc}$ sulfated-derivatives. $\mathrm{IC}_{50}$, extracted from SPR derived inhibitions curves, are presented as a function of the GlcNAc sulfation pattern and for a given version of Langerin (see SI appendix, Material and Methods for corresponding sensorgrams and inhibitory curves). (*) For GlcNS6S, the $\mathrm{IC}_{50}$ for DC-SIGN has not been reached experimentally (see from (B)) and thus extrapolated from the inhibition curve equation.

The different sulfated versions of $N$-Acetyl-D-glucosamine evaluated for their relative affinity $\left(\mathrm{IC}_{50}\right)$ for DC-SIGN and Langerin are presented in Figure 2A, Figure 2B, and Figure 2C. Non-sulfated GlcNAc has the same affinity for both lectins (5 mM, Figure 2C) and comparable with the reported affinity of Mannose for DC-SIGN. ${ }^{21}$ However, adding 
one or several negative sulfate groups onto the ligand results in an increase in affinity in favor of Langerin, while recognition by DC-SIGN drastically decreases. This results in a selectivity factor of 5000 in favor of Langerin as measured for the disulfated ligand, GlcNS6S. Significantly, a single sulfation at position 6 of GlcNAc is responsible for a selectivity factor close to 600 ( $\mathrm{IC}_{50}$ of $0.38 \mathrm{mM}$ and $0.22 \mathrm{mM}$ for GlcNAc6S and GlcNS6S respectively).

Recognition mechanism of sulfated ligands. The interaction of sulfated saccharides is a unique feature of Langerin compared to DC-SIGN. To understand determinants of this specificity, we analyzed the affinities of sulfated ligands with two active site mutants of Langerin-extracellular domain (Lg-ECD). We studied the roles of lysines 299 and 313 (Figure 1A), which are ideally positioned in the $\mathrm{Ca}^{2+}$ site surroundings. Figure 3 summarizes the $\mathrm{IC}_{50}$, determined by SPR inhibition assay, for each ligand as a function of the Lg-ECD mutations. Lysine mutations induce an overall decrease in affinity of the sulfated ligand for Langerin (Figure 2D). Mutation K313A completely abolishes the improved affinity observed upon sulfation of the ligands. In addition, the K299A mutation leads to a two-fold reduction in GlcNAc6S ligand $\mathrm{IC}_{50}$ when compared to wild type Langerin. This effect is more pronounced for the K313A mutant, with a decrease of the affinity of GlcNAc6S for Langerin by a factor of 10. The key role of lysine 313 is also observed in the crystallographic structure of Lg-carbohydrate recognition domain (CRD) with GlcNS6S.

$\mathrm{X}$-ray crystal structure of Lg-CRD/GIcNS6S complex. The crystal contains four copies of the CRD per asymmetric unit within a $\mathrm{P}_{2}$ space group. The structure solved at $1.89 \AA$ is well conserved when compared to previously determined structures of Lg-CRD as illustrated by RMSD of $0.443 \AA, 0.335 \AA$ and $0.331 \AA$ with Lg-CRD structures without ligand or complexed with $6 \mathrm{SO}_{4}-\mathrm{Gal} 11-4 \mathrm{GlcNAC}$ or Man $\alpha 1-2 \mathrm{Man}$ (pdb codes are 3c22, 3p5i and 3p5f, respectively). ${ }^{37,40}$ As structure resolution was conducted by molecular replacement using an unliganded Lg-CRD model, the identification of a well-defined electron density onto the $\mathrm{Ca}^{2+}$ ion allowed to clearly establish the presence of the GlcNS6S ligand within the binding site (Figure 3A). As expected, GlcNS6S binds to the $\mathrm{Ca}^{2+}$ ion with its equatorials 3-OH and 4-OH groups. The anomeric $\mathrm{OH}$ group interacts with K299. Pertaining to the sulfate groups, the 6-sulfate is involved in an electrostatic interaction with $\mathrm{K} 313$, and the $\mathrm{N}$-sulfate in $\mathrm{C}_{2}$ is oriented towards the solvent (Figure 3B). In addition, three water molecules connect the ligand to the protein (see Supporting Information, Figure $\mathrm{S} 1)$.
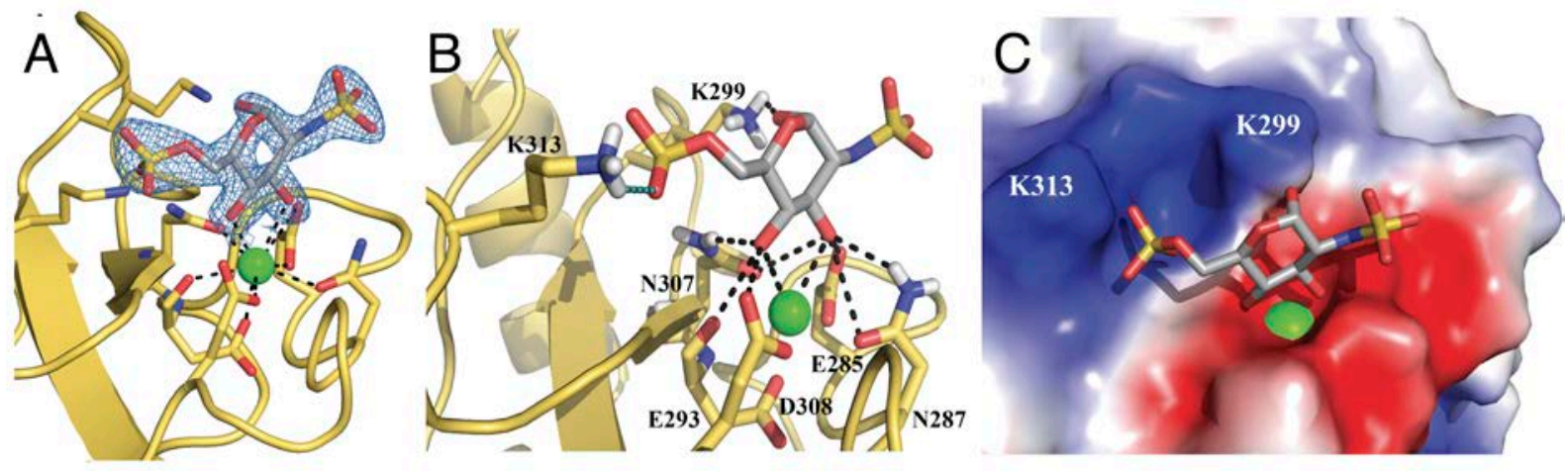

Figure 3. Binding mode of GlcNS6S within Langerin calcium binding site. (A) Electron density map for bound GlcNS6S. The bound ligand is shown superimposed on the Fo-Fc electron density map contoured at $3 \sigma$. Bonds involved in $\mathrm{Ca}^{2+}$ binding are in black dashed line. (B) Interaction network involved in GlcNS6S interaction. Black dashed line for polar interaction and green dashed light for ionic bond. (C) Electrostatic potential of Lg-CRD surface in the Lg-CRD/GlcNS6S complex highlight the positive area around K313 and K299 positions.

It appears that K299 have a minor role in the binding whereas the K313 plays a preponderant role in the affinity of 6sulfated ligands (Figure 3C), in agreement with the impact of K313A mutation reported above. However, both lysines contribute to the establishment of a strong positive electrostatic region, adjacent to the $\mathrm{Ca}^{2+}$ binding site, that allows to accommodate diverse sulfated glycans (Figure $3 C$ ). For instance, in the binding mode of $6 \mathrm{SO}_{4}-\mathrm{Gal}_{3} 1-4 \mathrm{GlcNac}$, the 
sulfate group of Gal6S was clearly oriented between K313 and K299. In that case, the two lysines were both essential to the sulfate recognition. ${ }^{37,41,42}$ Recently, we have characterized the binding mode to Lg-CRD of 8 heparin-derived trisaccharides, composed of GlcN-IdoA-GlcN, with different sulfation patterns. ${ }^{39}$ In all cases, it is the terminal nonreducing GlcN that is binding to the $\mathrm{Ca}^{2+}$ ion. The IdoA and GlcN residues extend between K313 and K299 and establish secondary contacts with Langerin surface. Here, it emerges that these two lysines are dynamic in their interactions with the sulfate group. Notably, K313 can shift to accommodate various sulfated ligands (see SI appendix Figure S2).

Aiming to develop a highly specific antagonist of DC-SIGN versus Langerin, the observation that a negative charge on the sugar $\mathrm{C}_{6}$ specifically interacts with $\mathrm{K} 313$ of Langerin binding site represents the starting-point for a glycomimetic design strategy. The X-ray structure of DC-SIGN complex with glycomimetic $\mathbf{1}^{23}$ shows that the $\mathrm{C}_{6}$ position of the sugar unit is not involved in any direct interaction (Figure 1C) and it is located in a very open area, close to a negatively charged patch of DC-SIGN surface. A positive charge and/or a steric hindrance in position $\mathrm{C}_{6}$ of 1 should lead to an electrostatic repulsion or steric clashes with K313 within Langerin binding site. Glycomimetics modified in position $\mathrm{C}_{6}$ are expected to differentially impact binding to Langerin and to DC-SIGN.

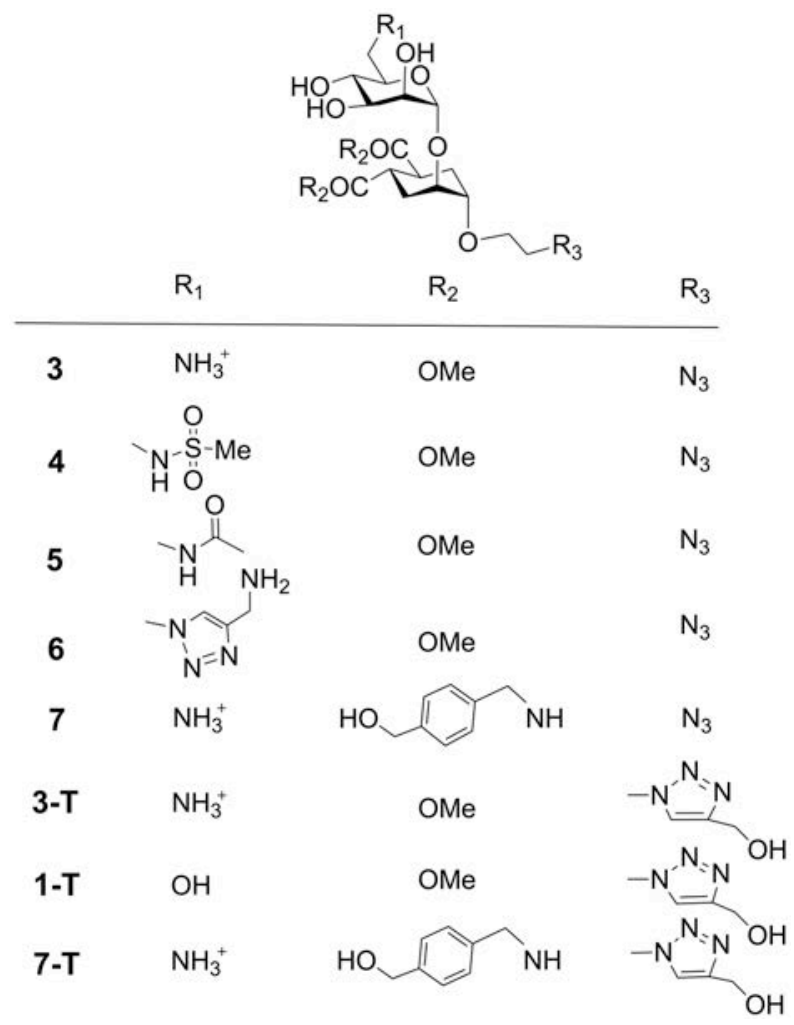

Figure 4. Structure of 6-modified pseudo-dimannosides 3-7, and of some triazole derivatives (-T).

Synthesis and screening of 6-modified compound 1 library towards DC-SIGN and Langerin. Based on the lead compound 1, we synthesized a novel library of four compounds modified at position 6 of the mannose ring (compounds 3 to 6, Figure 4). SPR competition studies between compound 1 and its 6-amino counterpart, compound 3 (Table 1), clearly showed a strong enhancement of DC-SIGN selectivity in compound $\mathbf{3}$.

While the selectivity is a modest 1.54 for the initial lead $\mathbf{1}$, compound $\mathbf{3}$ reaches a selectivity factor better than 7 . We also observed that the relative affinity for DC-SIGN was improved $\left(\mathrm{IC}_{50}=481 \mu \mathrm{M}\right)$, compared to the lead compound 1 $\left(\mathrm{IC}_{50}=956 \mu \mathrm{M}\right)$. Thus, as expected, the positively charged amino group results in an improvement of DC-SIGN affinity and selectivity. 
Table 1: Evaluation of glycomimetics inhibition potency towards DC-SIGN and Langerin (SPR). n.a. not applicable, n.d. not determined.

\begin{tabular}{|c|c|c|c|c|c|}
\hline \multirow[t]{2}{*}{ Compounds } & \multicolumn{2}{|c|}{ IC50 $(\mu \mathrm{M})$} & \multirow[t]{2}{*}{ Selectivity } & \multicolumn{2}{|c|}{ Inhibition (\%) at $4.4 \mathrm{mM}$} \\
\hline & DC-SIGN & Langerin & & DC-SIGN & Langerin \\
\hline Man1-2Man & $915 \pm 25$ & $1680 \pm 61$ & 1.83 & 89.8 & 74.6 \\
\hline 1 & $956 \pm 38$ & $1474 \pm 329$ & 1.54 & 83.3 & 40.8 \\
\hline 2 & $329 \pm 5$ & $2556 \pm 610$ & 7.76 & 99.6 & 36.3 \\
\hline 3 & $481 \pm 11$ & $3440 \pm 668$ & 7.15 & 97.3 & 41.9 \\
\hline 4 & $703 \pm 29$ & $1236 \pm 215$ & 1.75 & n.d. & n.d. \\
\hline 5 & $1235 \pm 40$ & $1933 \pm 90$ & 1.56 & n.d. & n.d. \\
\hline 6 & $1366 \pm 81$ & $4035 \pm 145$ & 2.95 & n.d. & n.d. \\
\hline 7 & $254 \pm 5$ & no inhib. & n.a. & 99.7 & 0 \\
\hline
\end{tabular}

Merging modifications from different optimized glycomimetics. Compound $\mathbf{2}$ had been previously selected as a first optimization of $\mathbf{1}$, on the basis of its relative DC-SIGN affinity (three times improvement of the $\mathrm{IC}_{50}$ ). ${ }^{26} \mathrm{The}^{2}$ Langerin screening reported in Table 1 shows an unexpected selectivity improvement of $\mathbf{2}$ over $\mathbf{1}$ by a factor of 7 . The binding mode of 2 in DC-SIGN, characterized by STD NMR (Figure 1D), ${ }^{26}$ is conserved and the additional hydroxymethylene-benzyl groups increase the surface contact of the ligand within the DC-SIGN binding site. As for $\mathbf{1}$, this binding mode should not be impaired by modifications in position 6 of the mannose moiety.
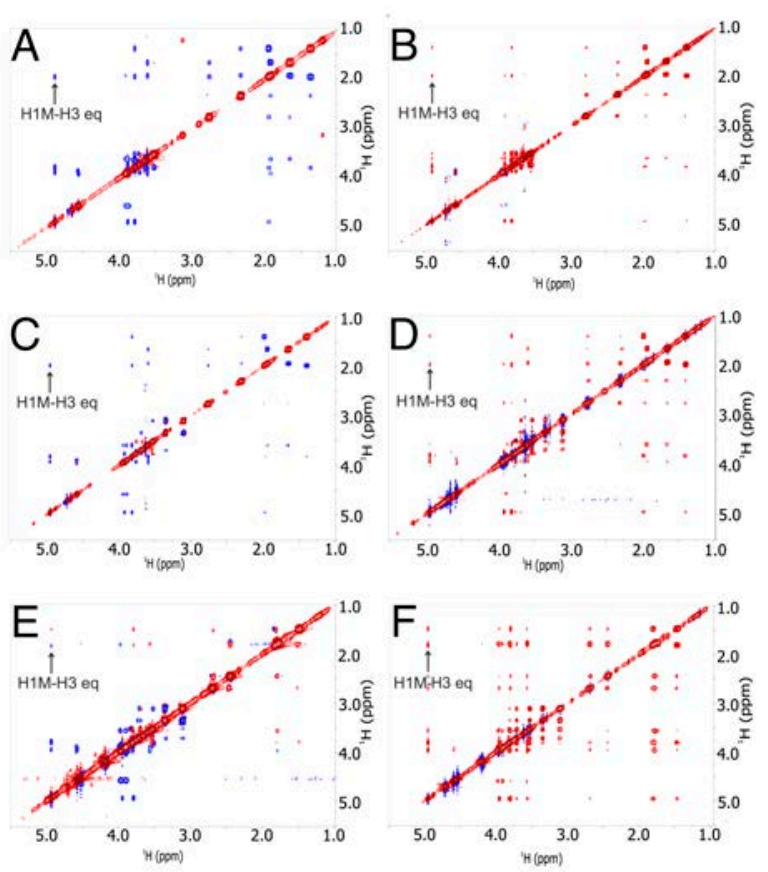

Figure 5. NOESY $((\mathbf{A}),(\mathbf{C}),(\mathbf{E}))$ and transfer NOESY $((\mathbf{B}),(\mathbf{D}),(\mathbf{F}))$ experiments for 3-T $((\mathbf{A})$ and $(\mathbf{B}))$, 1-T $((\mathbf{C})$ and $(\mathbf{D}))$, and 7-T $((\mathbf{E})$ and $(\mathbf{F}))$. Cross-peaks corresponding with the NOE between Mannose anomeric proton H1M and H3 equatorial of cyclohexyl are labelled. Peaks belonging to the central cyclohexyl moiety of 7-T are negative contrasting with 3-T and 1-T (see (E)). This behaviour corresponds to a large molecule, and is consequence of the introduction of two additional benzyl groups.

To verify this hypothesis, we have analysed the effect of the 6-amino group in the binding to DC-SIGN using NMR transient methods (STD-NMR and NOESY experiments for the complex and the free mimic). ${ }^{43}$ Previously, it was shown that a triazole-modified compound $\mathbf{1}$ analog (as in 1-T, Figure 4) does not interfere or influence the binding and 
the geometry of the bound conformation remains the same. ${ }^{26}$ NOESY and tr-NOESY analysis of the triazole derivative of 3, show that, both in the free state and in the protein complex, 3-T maintains the same conformation as $\mathbf{1}$ and $\mathbf{2}$, i.e. an extended conformation. An exclusive NOE between H1 of Mannose (H1M) and -H3eq of the cyclohexyl ring is shown on Figure 5. Additionally, the same pattern and intensity of STD signals (see SI appendix) was obtained for 3-T and 1-T (saturation time 3s), strongly suggesting that the two molecules share a common binding mode with DCSIGN.

Thus, we synthesized a third-generation glycomimetic, compound 7, combining the two modifications of the secondgeneration compounds 2 and 3 (Figure 4). A quantitative STD-NMR analysis of 7-T was frustrated by spectral congestion. Attempts to solve the signal overlap by including a second ${ }^{1} \mathrm{H}-{ }^{13} \mathrm{C}$ transference after the STD module using an HSQC to spread the peaks along a second dimension were unsuccessful due to low signal. Thus, for the study of the DC-SIGN interaction of 7-T we rely only on the transfer NOE data reflecting the structural properties of the bound conformation. Transfer NOESY experiments clearly showed the exclusive NOE of the extended conformation as for 3T (Figure 5), consequently we can assess that bound conformations of 3-T and 7-T are equivalent.

SPR competition tests showed no major alteration of the affinity of $\mathbf{7}$ for DC-SIGN relative to $\mathbf{2}$ and $\mathbf{3}$ (Table 1, DCSIGN IC $\left.5_{50} 254 \mu \mathrm{M}\right)$. However, the cumulative modifications from $\mathbf{2}$ and $\mathbf{3}$ as assembled in $\mathbf{7}$ appeared to induce a drastic synergistic effect on selectivity against Langerin. Indeed, no $\mathrm{IC}_{50}$ for 7 could be determined in the competitionLangerin binding assay, since no inhibition was observed in the concentration range (up to $4.4 \mathrm{mM}$ ). Thus, no selectivity factor could be calculated. Gratifyingly, this data suggests that binding to Langerin is excluded for 7.

To further illuminate this finding, we evaluated the level of binding inhibition for both DC-SIGN and Langerin using the maximal concentration sustainable in the assay $(4.4 \mathrm{mM})$ with ligands from the natural ligand Mana1-2Man to the mimics 1, 2, 3 and 7 (Table 1). At this high concentration, compounds 2 and 3 inhibit Langerin 36.3\% and 41.9\%, respectively, while the DC-SIGN binding inhibition reaches almost 100\% for both ligands. Remarkably, compound 7 results in almost 100\% inhibition of DC-SIGN binding with no observed Langerin inhibition.

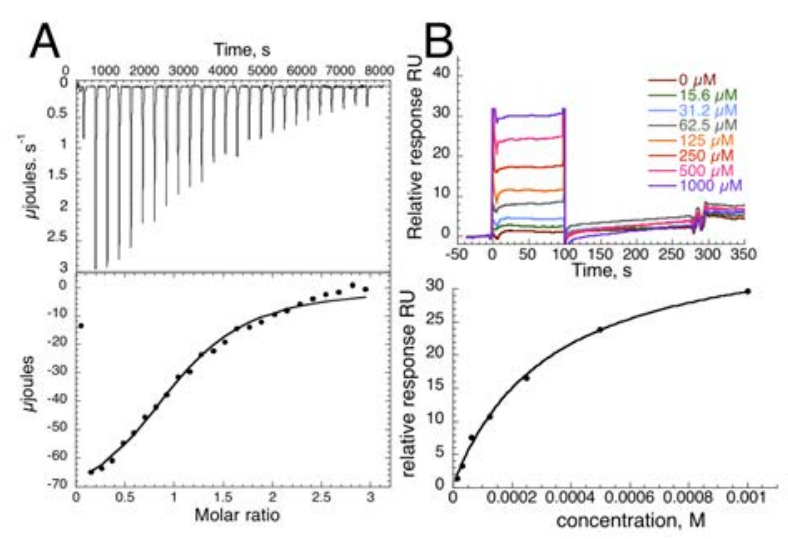

Figure 6: ITC and SPR analysis of glycomimetic 7 titration to DC-SIGN ECD. (A) Titration of glycomimetic 7 at 4.7 $\mathrm{mM}$ to DC-SIGN ECD $(566 \mu \mathrm{M})$. Upper panel shows the titration thermogram, and lower panel the data integration with fitted curves (1:1 binding model). (B) Titration of glycomimetic 7 onto DC-SIGN ECD functionalized surface. Upper panel shows reference-subtracted sensorgrams of increasing concentrations of 7 and lower panel the steady state binding analysis (1:1 binding model).

Characterizations of compound 7 binding properties to DC-SIGN. We further analyzed the interaction properties of 7 with DC-SIGN ECD by ITC. The pseudosaccharide $7(4.7 \mathrm{mM})$ was titrated into lectin solution $(566 \mu \mathrm{M})$. The ITC data of the titration of 7 to DC-SIGN ECD confirm the moderate affinity (Figure 6A). Indeed, the titration curve did not adopt the full sigmoidal shape. Fitting one binding site model to the data with an assumed stoichiometry value n fixed to 1 yielded the $K_{d}$ of $171 \pm 11 \mu \mathrm{M}$, which was in the same range as the apparent affinity determined by SPR 
competition assay $\left(\mathrm{IC}_{50} 254 \mu \mathrm{M}\right)$. So far, the combination of the moderate affinity and a low molecular weight of these glycomimetics, have precluded the evaluation of a $K_{d}$ by SPR in a direct interaction mode. Besides, improvement of the affinity, below $\mathrm{mM}$ range, allows to observe in real time the glycomimetic binding on a DC-SIGN ECD functionalized surface. The resulting $\mathrm{K}_{d}$ is $310 \mu \mathrm{M}$ for compound 7 (Figure 6B). From these different and complementary analyses, an affinity in the range of $250 \mu \mathrm{M}$ can be assumed for 7 . The ITC analysis yielded a $\Delta \mathrm{H}=$ $-9.54 \pm 0.26 \mathrm{~kJ} \mathrm{~mol}^{-1}$ and a $\mathrm{T} \Delta \mathrm{S}=11.9 \mathrm{~kJ} \cdot \mathrm{mol}^{-1}$, leading to a $\Delta \mathrm{G}$ of $-21.4 \mathrm{~kJ} \cdot \mathrm{mol}^{-1}$. Thus the binding mode of 7 is almost equally enthalpically and entropically driven.

CLRs are involved in numerous aspects of immune regulation and they have attracted great interest as targets for antiinfective strategies, vaccine delivery, immune modulation, etc. However, due to their large open binding sites, their low affinity for their natural ligands and the carbohydrate cross-recognition between some CLRs, the number of specific ligands is limited. Indeed, when considering only computed druggability scores, most CLRs were predicted to be challenging or even undruggable targets. ${ }^{44}$ Despite this challenging task, recent progress has been made towards defining potent antagonists against CLRs, such as DC-SIGN, Mincle, and DCIR. ${ }^{29,45,46}$ However, we are still far from compounds with the required affinity and the relevant specificity to envision their use in clinical trials.

To address specific difficulties encountered regarding selectivity, we defined, in this work, a new strategy to improve our rationally-designed glycomimetics. DC-SIGN and Langerin are CLRs for which development of a highly specific antagonist would be of great potential impact. ${ }^{47}$ The rational design of compounds selective for DC-SIGN is very challenging due to its very open binding site which is difficult to exploit (Figure 1C). Thus, instead of modifying the ligand by adding additional anchoring points within the targeted binding site, as in classical approaches, we searched for optimizations within our compounds that could impair their interaction within the Langerin binding site. In order to do this, we first made a careful comparative analysis of the binding mode of natural ligands known to be specific for one of the two CLRs. Having defined the differential determinants between the CLR binding sites and their natural ligands, we can more completely understand the source of ligand selectivity. Indeed, we showed that residue K313 in the Langerin binding site strongly interacts with a sulfate group at $\mathrm{C}_{6}$ of carbohydrate ligands. Then we exploited this finding to introduce modifications that should selectively alter Langerin recognition, while maintaining tight DC-SIGN binding. Exploiting the differences between both CLR binding sites and rational designed ligand modifications, this strategy succeeded in generating a highly specific compound for DC-SIGN. Following the evolution of $\mathbf{1}$ to 2 and $\mathbf{3}$ and finally to 7, we have improved the selectivity with compound 7 to such a level that neither the interaction nor the selectivity factor with Langerin are measurable. We can target DC-SIGN specificity rather than selectivity with the new compound developed in this work. This strategy, where ligand improvements can be obtained through differential analysis between two competing receptors for a given ligand, can be of general use in the development of inhibitors. This approach is of particular interest when improvements in ligand/target affinity are limited by biophysical phenomena, as in the case of CLRs. Once the selectivity is achieved, the increase of affinity can be then reached by avidity effect through multivalent displaying of glycomimetics.

\section{Materials and Methods}

Compounds. The GlcNAc-sulfated derivatives (Figure 2A) were purchased from Dextra Laboratories.

DC-SIGN and Langerin ECD production and purification. WT and mutated recombinant forms of DC-SIGN and Langerin extracellular domain (DC-SIGN ECD and Lg-ECD respectively) were produced and purified as described previously. ${ }^{40,48}$

Crystallization. Crystals of Lg-CRD were grown from a sample of Lg-ECD by the hanging-drop vapor-diffusion method at $293 \mathrm{~K}$ with a protein/reservoir drop ratio of $1: 1$, at a protein concentration of $15 \mathrm{mg} / \mathrm{mL}$ in $25 \mathrm{mM} \mathrm{Tris-HCl}$ 
$\mathrm{pH} 8,150 \mathrm{mM} \mathrm{NaCl}$ and $4 \mathrm{mM} \mathrm{CaCl}_{2}$ and a reservoir containing $100 \mathrm{mM}$ HEPES pH 7, $100 \mathrm{mM} \mathrm{MgCl}_{2}, 150 \mathrm{mM}^{2}$ $\mathrm{NaCl}$ and 20\% PEG 3350 supplemented or not with $70 \mathrm{mM}$ europium dipicolinate. The use of this lanthanide complex was motivated by the aim to crystallize the full-length Langerin ECD protein by inducing interactions between the complex and arginine residues to promote crystal contacts. ${ }^{49}$ Crystals appeared after 2 months with the typical LgCRD crystal shape and dimension described previously. ${ }^{50}$ In drop proteolysis of Lg-ECD, by residual protease, between the CRD and the Neck domain of Langerin account for this phenomenon.

This was confirmed by evaluation of the space group and the cell dimension. For Lg-CRD/GlcNS6S complex preparation, crystals were firstly washed in $100 \mathrm{mM}$ HEPES pH7, $100 \mathrm{mM} \mathrm{MgCl}_{2}, 150 \mathrm{mM} \mathrm{NaCl}$ and $35 \%$ PEG 3350 for the cryoprotection and secondly soaked and equilibrated by vapor diffusion during $1 \mathrm{~h}$ in $1 \mu \mathrm{L}$ of the previous solution supplemented with $10 \mathrm{mM}$ of GlcNS6S. Crystals were directly flash frozen in liquid nitrogen.

Data collection, processing and structure determination. X-ray diffraction data were collected in the BM30a beamline at the ESRF Grenoble using an ADSC Q315r detector. The crystals was diffracted with a wavelength of $0.979 \AA$, exposition time of 20 sec/images, 200 images with an angular step of 0.5 degree and a detector distance of $220 \mathrm{~mm}$. Data processing is described in SI appendix, Structural analysis. Coordinates and associated structure factors have been deposited in the PDB database, code: $5 \mathrm{~g} 6 \mathrm{u}$.

Surface Plasmon Resonance analysis. Surface plasmon resonance experiments were performed on a Biacore 3000 using a CM4 chip, functionalized at $5 \mu \mathrm{L} / \mathrm{min}$. Fc1 anf Fc3 was prepared as reference surface. Flow cell (Fc) from 1 to 4 were activated with $50 \mu \mathrm{L}$ of a $0.2 \mathrm{M} \mathrm{EDC/} 0.05 \mathrm{M}$ NHS mixture. After this step, Fc1 to Fc4 were respectively functionalized with bovine serum albumine (BSA) and mannosylated bovine serum albumine (BSA-Man, BSAman 1 1-3[mana1-6]man, Dextra laboratories, $60 \mu \mathrm{g} \cdot \mathrm{mL}^{-1}$ ). Then remaining activated groups of both cells were blocked with $30 \mu \mathrm{L}$ of $1 \mathrm{M}$ ethanolamine. After blocking, the four $\mathrm{Fc}$ were treated with $5 \mu \mathrm{L}$ of $10 \mathrm{mM} \mathrm{HCl}$ to remove unspecific bound protein and $5 \mu \mathrm{L}$ of $50 \mathrm{mM}$ EDTA to expose surface to regeneration protocol. Finally, $238 \mathrm{RU}$ and 1847 RU of BSA and BSA-Man were respectively immobilized on Fc1 and Fc2.

For inhibition studies, on Fc2 or Fc4, $20 \mu \mathrm{M}$ of DC-SIGN ECD or $15 \mu \mathrm{L}$ of Langerin ECD respectively are mixed with increasing concentrations of inhibiting compounds were prepared in a running buffer composed of $25 \mathrm{mM}$ Tris pH8, $150 \mathrm{mM} \mathrm{NaCl}, 4 \mathrm{mM} \mathrm{CaCl}_{2}, 0.005 \%$ P20 surfactant, and $13 \mu \mathrm{L}$ of each sample was injected onto the surfaces at a $5 \mu \mathrm{L} /$ min flow rate. The resulting sensorgrams were reference surface corrected.

$$
y=R_{h i}-\frac{R_{h i}-R_{l o}}{1+\left(\frac{\text { Conc }}{A_{1}}\right)^{A_{2}}} \quad I C_{50}=A_{1} \cdot\left(\left(\frac{R_{h i}-R_{l o}}{R_{h i}-50}\right)^{\frac{1}{A_{2}}}-1\right)_{(2)}
$$

The DC-SIGN binding responses were extracted from sensorgrams, converted to percent residual activity values (y), which were plotted against corresponding compound concentration. The 4-parameter logistic model (equation 1) was fitted to the plots, and the $\mathrm{IC}_{50}$ values were calculated, from equation 2, using the values of fitted parameters $\left(\mathrm{R}_{\mathrm{hi}}, \mathrm{R}_{\mathrm{lo}}\right.$, $\mathrm{A}_{1}$ and $\mathrm{A}_{2}$ ).

Isothermal Titration Calorimetric analysis. ITC experiments were performed at $25{ }^{\circ} \mathrm{C}$ using TA Instrument Nano Isothermal Titration Calorimeter Low Volume (Nano ITC LV) with $190 \mu \mathrm{L}$ cell volume. Compound 7 and DC-SIGN ECD were prepared in $25 \mathrm{mM}$ Tris pH8, $150 \mathrm{mM} \mathrm{NaCl}, 4 \mathrm{mM} \mathrm{CaCl}_{2}$. Compound was stepwise injected (1.03 $\left.\mu \mathrm{L}\right)$ to DC- SIGN solution using 5 min intervals between injections. $566 \mu \mathrm{M}$ of monomeric DC-SIGN ECD and $0.47 \mathrm{mM}$ compound concentrations were used. The blank titrations (compounds to buffer) were done for subtraction of dilution heat from the integrated data. A one-site binding model was fit to the data (nanoAnalyse 2.20 TA), yielding association constants $\left(\mathrm{K}_{\mathrm{A}}\right)$ and binding enthalpies $(\Delta \mathrm{H})$. The free energy changes $(\Delta \mathrm{G})$ and entropy $(\Delta \mathrm{S})$ were calculated using equation: 
$\Delta \mathrm{G}=\Delta \mathrm{H}-\mathrm{T} \Delta \mathrm{S}=-\mathrm{RT} \ln \mathrm{K}_{\mathrm{A}}$, where $\mathrm{T}$ is the absolute temperature and $\mathrm{R}=8.314 \mathrm{~J} \cdot \mathrm{mol}^{-1} \cdot \mathrm{K}^{-1}$.

Synthesis. The 6-modified pseudo-disaccharides 3-7 were synthesized starting from 6-azidomannose tetra- $O$-benzoate following procedures previously established for $\mathbf{1}$ and 2. Full details are collected in the Supplementary Information file.

NMR analysis. All NMR experiments were performed on a Bruker Avance DRX $500 \mathrm{MHz}$ spectrometer equipped with a $5 \mathrm{~mm}$ inverse triple-resonance probe. All the samples were dissolved in $550 \mu \mathrm{L}$ of buffer $\mathrm{D}_{2} \mathrm{O}(150 \mathrm{mM} \mathrm{NaCl}, 4$ $\mathrm{mM} \mathrm{CaCl}_{2}, 25 \mathrm{mM}$ d-Tris, pD 7.2) with a ligand concentration of $2 \mathrm{mM}$ for free ligand analysis. For the experiments in the presence of receptor 19 or $9.5 \mu \mathrm{M}$ of DC-SIGN and $1.0 \mathrm{mM}$ of the ligand were used. The pD was adjusted to 7.2 in all samples. STD NMR experiments were carried out at $25{ }^{\circ} \mathrm{C}$. For protein saturation a train of Gaussian shaped pulses with a length of $49 \mathrm{~ms}$, an interpulse delay of $1 \mathrm{~ms}$ and an attenuation of $50 \mathrm{~dB}$ was used. The unwanted broad resonance signals of the protein were removed by use of a spin lock pulse of $15 \mathrm{~ms}$ prior to acquisition. For suppression of the water signal the WATERGATE scheme (3-9-19) was employed. The on-resonance frequency was set to $-1 \mathrm{ppm}$, whereas the off-resonance frequency was set to $40 \mathrm{ppm}$, interleaved recorded. Reference experiments were carried out to confirm the absence of direct irradiation of the ligand. Saturation times to obtain the build-up curves were $0.5,1,1.5,2,3$ and $4 \mathrm{~s}$. Additional experimental details are given in the Supplementary information file.

\section{ASSOCIATED CONTENT}

The supporting information is available free of charge on the ACS Publications website at DOI: XXXXX.

\section{AUTHOR INFORMATION}

Corresponding Authors

*E-mail: franck.fieschi@ibs.fr.

*E-mail: anna.bernardi@unimi.it.

\section{NOTES}

The authors declare no conflict of interest.

\section{ACKNOWLEDGMENT}

This work used the platforms of the Grenoble Instruct center (ISBG; UMS 3518 CNRS-CEA-UGA-EMBL). SPR and MP3 platforms support from FRISBI (ANR-10-INSB-05-02) and GRAL (ANR-10-LABX-49-01) within the Grenoble Partnership for Structural Biology (PSB). This work was supported with funds from CM1102 COST Action and EU ITN Marie Curie program Immunoshape (Grant no. 642870). We are grateful to EU ITN Marie-Curie program (CARMUSYS - Grant no. 213592) for funding Ieva Sutkeviciute and Norbert Varga. V. Porkolab was supported by la Région Rhône-Alpes. The authors thank to Cristina Solera for NMR spectra acquisition and Sebastian Temme for critical review of the manuscript.

\section{REFERENCES}

(1) van Vliet, S. J., García-Vallejo, J. J., and van Kooyk, Y. (2008) Dendritic cells and C-type lectin receptors: coupling innate to adaptive immune responses. Immunol Cell Biol 86, 580-587. 
(2) Bell, D., Young, J. W., and Banchereau, J. (1999) Dendritic cells. Adv Immunol 72, 255-324.

(3) van Kooyk, Y., Engering, A., Lekkerkerker, A. N., Ludwig, I. S., and Geijtenbeek, T. B. (2004) Pathogens use carbohydrates to escape immunity induced by dendritic cells. Curr Opin Immunol 16, 488-493.

(4) Geijtenbeek, T. B., van Vliet, S. J., Engering, A., t Hart, B. A., and van Kooyk, Y. (2004) Self- and nonselfrecognition by C-type lectins on dendritic cells. Annu. Rev. Immunol. 22, 33-54.

(5) Powlesland, A. S., Fisch, T., Taylor, M. E., Smith, D. F., Tissot, B., Dell, A., Pöhlmann, S., and Drickamer, K. (2008) A novel mechanism for LSECtin binding to Ebola virus surface glycoprotein through truncated glycans. J Biol Chem 283, 593-602.

(6) Zhao, D., Han, X., Zheng, X., Wang, H., Yang, Z., Liu, D., Han, K., Liu, J., Wang, X., Yang, W., Dong, Q., Yang, S., Xia, X., Tang, L., and He, F. (2016) The Myeloid LSECtin Is a DAP12-Coupled Receptor That Is Crucial for Inflammatory Response Induced by Ebola Virus Glycoprotein. PLoS Pathog 12, e1005487.

(7) Geijtenbeek, T. B., Kwon, D. S., Torensma, R., van Vliet, S. J., van Duijnhoven, G. C., Middel, J., Cornelissen, I. L., Nottet, H. S., KewalRamani, V. N., Littman, D. R., Figdor, C. G., and van Kooyk, Y. (2000) DC-SIGN, a dendritic cell-specific HIV-1-binding protein that enhances trans-infection of T cells. Cell 100, 587-597.

(8) Geijtenbeek, T. B., van Vliet, S. J., Koppel, E. A., Sanchez-Hernandez, M., Vandenbroucke-Grauls, C. M., Appelmelk, B., and van Kooyk, Y. (2003) Mycobacteria target DC-SIGN to suppress dendritic cell function. $J$ Exp Med 197, 7-17.

(9) Cambi, A., Gijzen, K., de Vries, J. M., Torensma, R., Joosten, B., Adema, G. J., Netea, M. G., Kullberg, B. J., Romani, L., and Figdor, C. G. (2003) The C-type lectin DC-SIGN (CD209) is an antigen-uptake receptor for Candida albicans on dendritic cells. Eur J Immunol 33, 532-538.

(10) Ernst, B., and Magnani, J. L. (2009) From carbohydrate leads to glycomimetic drugs. Nat Rev Drug Discov 8, 661-677.

(11) Sattin, S., and Bernardi, A. (2016) Glycoconjugates and Glycomimetics as Microbial Anti-Adhesives. Trends in Biotechnology 34, 483-495.

(12) Anderluh, M., Jug, G., Svajger, U., and Obermajer, N. (2012) DC-SIGN antagonists, a potential new class of antiinfectives. Curr. Med. Chem. 19, 992-1007.

(13) Borrok, M. J., and Kiessling, L. L. (2007) Non-carbohydrate inhibitors of the lectin DC-SIGN. J Am Chem Soc $129,12780-12785$.

(14) Garber, K. C. A., Wangkanont, K., Carlson, E. E., and Kiessling, L. L. (2010) A general glycomimetic strategy yields non-carbohydrate inhibitors of DC-SIGN. Chem Commun (Camb) 46, 6747-6749.

(15) Obermajer, N., Sattin, S., Colombo, C., Bruno, M., Svajger, U., Anderluh, M., and Bernardi, A. (2011) Design, synthesis and activity evaluation of mannose-based DC-SIGN antagonists. Mol. Divers. 15, 347-360.

(16) Andreini, M., Doknic, D., Sutkeviciute, I., Reina, J. J., Duan, J., Chabrol, E., Thépaut, M., Moroni, E., Doro, F., Belvisi, L., Weiser, J., Rojo, J., Fieschi, F., and Bernardi, A. (2011) Second generation of fucose-based DC-SIGN ligands : affinity improvement and specificity versus Langerin. Org Biomol Chem 9, 5778-5786.

(17) Becer, C. R., Gibson, M. I., Geng, J., Ilyas, R., Wallis, R., Mitchell, D. A., and Haddleton, D. M. (2010) Highaffinity glycopolymer binding to human DC-SIGN and disruption of DC-SIGN interactions with HIV envelope glycoprotein. J Am Chem Soc 132, 15130-15132.

(18) Martínez-Avila, O., Hijazi, K., Marradi, M., Clavel, C., Campion, C., Kelly, C., and Penadés, S. (2009) Gold manno-glyconanoparticles: multivalent systems to block HIV-1 gp120 binding to the lectin DC-SIGN. Chemistry 15, 9874-9888.

(19) Sattin, S., Fieschi, F., and Bernardi, A. (2015) DC-SIGN as a Target for Drug Development Based on Carbohydrates. In Carbohydrate Chemistry: State of the Art and Challenges for Drug Development (Cipolla, L., Ed), pp 379-394, Imperial College Presss, London.

(20) Reina, J. J., Sattin, S., Invernizzi, D., Mari, S., Martinez-Prats, L., Tabarani, G., Fieschi, F., Delgado, R., Nieto, P. M., Rojo, J., and Bernardi, A. (2007) 1,2-Mannobioside mimic: synthesis, DC-SIGN interaction by NMR and docking, and antiviral activity. ChemMedChem 2, 1030-1036.

(21) Sattin, S., Daghetti, A., Thépaut, M., Berzi, A., Sánchez-Navarro, M., Tabarani, G., Rojo, J., Fieschi, F., (null), and Bernardi, A. (2010) Inhibition of DC-SIGN-mediated HIV infection by a linear trimannoside mimic in a tetravalent presentation. ACS Chem Biol 5, 301-312.

(22) Berzi, A., Reina, J. J., Ottria, R., Sutkeviciute, I., Antonazzo, P., Sánchez-Navarro, M., Chabrol, E., (null), (null), Cetin, I., Rojo, J., Fieschi, F., Bernardi, A., and Clerici, M. (2012) A glycomimetic compound inhibits DC-SIGNmediated HIV infection in cellular and cervical explant models. AIDS 26, 127-137.

(23) Thépaut, M., Guzzi, C., Sutkeviciute, I., Sattin, S., Ribeiro-Viana, R., Varga, N., Chabrol, E., Rojo, J., Bernardi, A., Angulo, J., Nieto, P. M., and Fieschi, F. (2013) Structure of a Glycomimetic Ligand in the Carbohydrate Recognition Domain of C-type Lectin DC-SIGN. Structural Requirements for Selectivity and Ligand Design. $J$ Am Chem Soc 135, 2518-2529.

(24) Sutkeviciute, I., Thépaut, M., Sattin, S., Berzi, A., McGeagh, J., Grudinin, S., Weiser, J., Le Roy, A., Reina, J. J., Rojo, J., Clerici, M., Bernardi, A., Ebel, C., and Fieschi, F. (2014) Unique DC-SIGN clustering activity of a small glycomimetic: A lesson for ligand design. ACS Chem Biol 9, 1377-1385.

(25) Guzzi, C., Alfarano, P., Sutkeviciute, I., Sattin, S., Ribeiro-Viana, R., Fieschi, F., Bernardi, A., Weiser, J., Rojo, J., Angulo, J., and Nieto, P. M. (2016) Detection and quantitative analysis of two independent binding modes of a small ligand responsible for DC-SIGN clustering. Org Biomol Chem 14, 335-344.

(26) Varga, N., Sutkeviciute, I., Guzzi, C., McGeagh, J., Petit-Haertlein, I., Gugliotta, S., Weiser, J., Angulo, J., 
Fieschi, F., and Bernardi, A. (2013) Selective targeting of dendritic cell-specific intercellular adhesion molecule-3grabbing nonintegrin (DC-SIGN) with mannose-based glycomimetics: synthesis and interaction studies of bis(benzylamide) derivatives of a pseudomannobioside. Chemistry (Weinheim an der Bergstrasse, Germany) 19, 4786-4797.

(27) Mitchell, D. A., Jones, N. A., Hunter, S. J., Cook, J., Jenkinson, S. F., Wormald, M. R., Dwek, R. A., and Fleet, G. W. J. (2007) Synthesis of 2-C-branched derivatives of D-mannose: 2-C-aminomethyl-D-mannose binds to the human C-type lectin DC-SIGN with affinity greater than an order of magnitude compared to that of D-mannose. Tetrahedron: Asymmetry 18, 1502-1510.

(28) Tomašić, T., Hajšek, D., Svajger, U., Luzar, J., Obermajer, N., Petit-Haertlein, I., Fieschi, F., and Anderluh, M. (2014) Monovalent mannose-based DC-SIGN antagonists: targeting the hydrophobic groove of the receptor. Eur $J$ Med Chem 75, 308-326.

(29) Varga, N., Sutkeviciute, I., Ribeiro-Viana, R., Berzi, A., Ramdasi, R., Daghetti, A., Vettoretti, G., Amara, A., Clerici, M., Rojo, J., Fieschi, F., and Bernardi, A. (2014) A multivalent inhibitor of the DC-SIGN dependent uptake of HIV-1 and Dengue virus. Biomaterials 35, 4175-4184.

(30) Ordanini, S., Varga, N., Porkolab, V., Thépaut, M., Belvisi, L., Bertaglia, A., Palmioli, A., Berzi, A., Trabattoni, D., Clerici, M., Fieschi, F., and Bernardi, A. (2015) Designing nanomolar antagonists of DC-SIGN-mediated HIV infection: ligand presentation using molecular rods. Chem Commun (Camb) 51, 3816-3819.

(31) Bernardi, A., Jiménez-Barbero, J., Casnati, A., De Castro, C., Darbre, T., Fieschi, F., Finne, J., Funken, H., Jaeger, K.-E., Lahmann, M., Lindhorst, T. K., Marradi, M., Messner, P., Molinaro, A., Murphy, P. V., Nativi, C., Oscarson, S., Penadés, S., Peri, F., Pieters, R. J., Renaudet, O., Reymond, J.-L., Richichi, B., Rojo, J., Sansone, F., Schäffer, C., Turnbull, W. B., Velasco-Torrijos, T., Vidal, S., Vincent, S., Wennekes, T., Zuilhof, H., and Imberty, A. (2013) Multivalent glycoconjugates as anti-pathogenic agents. Chem. Soc. Rev. 42, 4709-4727.

(32) de Witte, L., Nabatov, A., Pion, M., Fluitsma, D., de Jong, M. A. W. P., de Gruijl, T., Piguet, V., van Kooyk, Y., and Geijtenbeek, T. B. H. (2007) Langerin is a natural barrier to HIV-1 transmission by Langerhans cells. Nat Med 13, $367-371$.

(33) de Witte, L., Nabatov, A., and Geijtenbeek, T. B. H. (2008) Distinct roles for DC-SIGN+-dendritic cells and Langerhans cells in HIV-1 transmission. Trends Mol Med 14, 12-19.

(34) de Jong, M. A. W. P., and Geijtenbeek, T. B. H. (2010) Langerhans cells in innate defense against pathogens. Trends in Immunology 31, 452-459.

(35) van den Berg, L. M., Ribeiro, C. M. S., Zijlstra-Willems, E. M., de Witte, L., Fluitsma, D., Tigchelaar, W., Everts, V., and Geijtenbeek, T. B. H. (2014) Caveolin-1 mediated uptake via langerin restricts HIV-1 infection in human Langerhans cells. Retrovirology 11, 3903.

(36) Galustian, C., Park, C. G., Chai, W., Kiso, M., Bruening, S. A., Kang, Y.-S., Steinman, R. M., and Feizi, T. (2004) High and low affinity carbohydrate ligands revealed for murine SIGN-R1 by carbohydrate array and cell binding approaches, and differing specificities for SIGN-R3 and langerin. Int Immunol 16, 853-866.

(37) Feinberg, H., Taylor, M. E., Razi, N., Mcbride, R., Knirel, Y. A., Graham, S. A., Drickamer, K., and Weis, W. I. (2011) Structural basis for langerin recognition of diverse pathogen and mammalian glycans through a single binding site. J Mol Biol 405, 1027-1039.

(38) Chabrol, E., Nurisso, A., Daina, A., Vassal-Stermann, E., Thépaut, M., Girard, E., Vivès, R. R., and Fieschi, F. (2012) Glycosaminoglycans are interactants of Langerin: comparison with gp120 highlights an unexpected calciumindependent binding mode. PLoS ONE (Karamanos, N. K., Ed.) 7, e50722.

(39) Muñoz-García, J. C., Chabrol, E., Vivès, R. R., Thomas, A., de Paz, J. L., Rojo, J., Imberty, A., Fieschi, F., Nieto, P. M., and Angulo, J. (2015) Langerin-heparin interaction: two binding sites for small and large ligands as revealed by a combination of NMR spectroscopy and cross-linking mapping experiments. J Am Chem Soc 137, 4100-4110.

(40) Thépaut, M., Valladeau, J., Nurisso, A., Kahn, R., Arnou, B., Vivès, C., Saeland, S., Ebel, C., Monnier, C., Dezutter-Dambuyant, C., Imberty, A., and Fieschi, F. (2009) Structural studies of langerin and Birbeck granule: a macromolecular organization model. Biochemistry 48, 2684-2698.

(41) Tateno, H., Ohnishi, K., Yabe, R., Hayatsu, N., Sato, T., Takeya, M., Narimatsu, H., and Hirabayashi, J. (2010) Dual Specificity of Langerin to Sulfated and Mannosylated Glycans via a Single C-type Carbohydrate Recognition Domain. Journal of Biological Chemistry 285, 6390-6400.

(42) Feinberg, H., Rowntree, T. J. W., Tan, S. L. W., Drickamer, K., Weis, W. I., and Taylor, M. E. (2013) Common polymorphisms in human langerin change specificity for glycan ligands. Journal of Biological Chemistry 288, 3676236771.

(43) Angulo, J., Ardá, A., Cabrita, E. J., Martin-Pastor, M., Jiménez-Barbero, J., and Nieto, P. M. (2015) NMR Techniques for the Study of Transient Intermolecular Interactions. In Structure Elucidation in Organic Chemistry (Bravo, M. M., Ed.), pp 325-360, Wiley-VCH, Weinheim, Germany.

(44) Aretz, J., Wamhoff, E.-C., Hanske, J., Heymann, D., and Rademacher, C. (2014) Computational and experimental prediction of human C-type lectin receptor druggability. Front Immunol 5, 323.

(45) Feinberg, H., Jégouzo, S. A. F., Rowntree, T. J. W., Guan, Y., Brash, M. A., Taylor, M. E., Weis, W. I., and Drickamer, K. (2013) Mechanism for recognition of an unusual mycobacterial glycolipid by the macrophage receptor mincle. Journal of Biological Chemistry 288, 28457-28465.

(46) Lambert, A. A., Azzi, A., Lin, S.-X., Allaire, G., St-Gelais, K. P., Tremblay, M. J., and Gilbert, C. (2013) Dendritic cell immunoreceptor is a new target for anti-AIDS drug development: identification of DCIR/HIV-1 inhibitors. PLOS ONE 8, e67873. 
(47) Wamhoff, E.-C., Hanske, J., Schnirch, L., Aretz, J., Grube, M., Varón Silva, D., and Rademacher, C. (2016) (19)F NMR-Guided Design of Glycomimetic Langerin Ligands. ACS Chem Biol 11, 2407-2413.

(48) Tabarani, G., Thépaut, M., Stroebel, D., Ebel, C., Vivès, C., Vachette, P., Durand, D., and Fieschi, F. (2009) DCSIGN neck domain is a $\mathrm{pH}$-sensor controlling oligomerization: SAXS and hydrodynamic studies of extracellular domain. J Biol Chem 284, 21229-21240.

(49) Pompidor, G., D'Aléo, A., Vicat, J., Toupet, L., Giraud, N., Kahn, R., and Maury, O. (2008) Protein Crystallography through Supramolecular Interactions between a Lanthanide Complex and Arginine. Angew Chem Int Ed Engl 47, 3388-3391.

(50) Thépaut, M., Vivès, C., Pompidor, G., Kahn, R., and Fieschi, F. (2008) Overproduction, purification and preliminary crystallographic analysis of the carbohydrate-recognition domain of human langerin. Acta Crystallogr $F$ Struct Biol Cryst Commun 64, 115-118. 